\title{
Recurrent Unresectable Aggressive Fibromatosis of Neck
}

\author{
Mohsin Raza ${ }^{1 *}$, Naila Zahid ${ }^{1}$, Saleha Anwar² and Syed Mehmood UI Haq ${ }^{3}$ \\ 'Department of Oncology, Liaquat National Hospital and Medical College, Karachi, Pakistan \\ 2Department of Radiology, Liaquat National Hospital and Medical College, Karachi, Pakistan \\ ${ }^{3}$ Department of Thoracic and Cardiac Surgery, Liaquat National Hospital and Medical College, Karachi, Pakistan
}

\begin{abstract}
Aggressive fibromatosis or desmoid tumors are classified as low grade, locally aggressive sarcomas with high propensity to recur and are lacking a clear census on management of recurrent-surgically in excisable tumors. We report here the case of a $27 y r s$ old male with aggressive fibromatosis that recurred following a surgical excision, and resulted in hampering the quality of life. The disease was managed using cytotoxic chemotherapy with doxorubicin, resulting in significant clinical and radiological response. It was followed by maintenance regimen using a less aggressive approach with non-steroidal anti-inflammatory drugs such as celecoxib that resulted in further clinical response at 2 months follow up. Therefore, the therapy was further continued.
\end{abstract}

Keywords: Fibromatosis, recurrent, chemotherapy, nsaids.

\section{INTRODUCTION}

Aggressive fibromatosis, also referred to as desmoid tumors, are mesenchymal neoplasms, frequently considered to be locally malignant but non-metastasizing tumors. The location and presentation of desmoids is variable, from abdominal wall, to intra-abdominal mesenteric masses, and to large extremity masses, which are generally seen in older patients of both genders. Mutations in the CTNNB1 gene encoding the $\beta$-catenin pathway have been identified in sporadic desmoid tumors, although the correlation of CTNNB1 mutation status with the clinical outcome remains uncertain [1-5]. The primary treatment for patients with resectable desmoid tumors is surgery [6-10]. With tumor lo-cation, size, patients' age, and margin status been identified as factors associated with recurrence following resection and Extra-abdominal tumors having a higher risk of recurrence than abdominal tumors.

\section{CASE PRESENTATION}

27 years old male patient presented in Oncology Outpatient Department with complains of recurrent right neck mass along with numbness and difficulty in griping \& holding in right hand over the last 2 months.

On examination there was a firm, non-tender mass just posterior to trachea up to anterior aspect of trapezius and inferiorly it was extending up to supraclavicular fossa on the right side. The ipsilateral winging of scapula and claw hand was appreciable on examination.

\section{PAST HISTORY}

Patient reported history of similar complains at ipsilateral site when he presented at an other health care set up

\footnotetext{
*Corresponding Author: Mohsin Raza, Department of Oncology, Liaquat National Hospital and Medical College, Karachi, Pakistan, Email:sinmoss@hotmail.com

Received: February 27, 2020; Revised: June 20, 2020; Accepted: July 03, 2020 DOI: https://doi.org/10.37184/lntbj.2708-7808.2.6
}

a year ago, where the imaging followed by resection of tumor was done, up to $95 \%$ tumor was excised and biopsied, and morphologically composed of spindle shaped cells with immunohistochemistry markers staining with beta-catenin, thus diagnosis of fibromatosis was made and the patient was advised a close follow up and surveillance, due to benign nature of disease.

The patient remained well for about 8 months and then due to recurrence of symptoms, he consulted thoracic surgery department from where the patient was referred in our clinic for opinion.

\section{RADIOLOGICAL DETAILS}

On presentation, the patient's MRI neck study with contrast was requested reveling the redemonstrations of abnormal signal intensity mass measuring about $13 \times 6.8 \times 10 \mathrm{~cm}$ in the right lower neck with extension into right hemithorax, with lobulated margins (Figs. 1A\&1B).

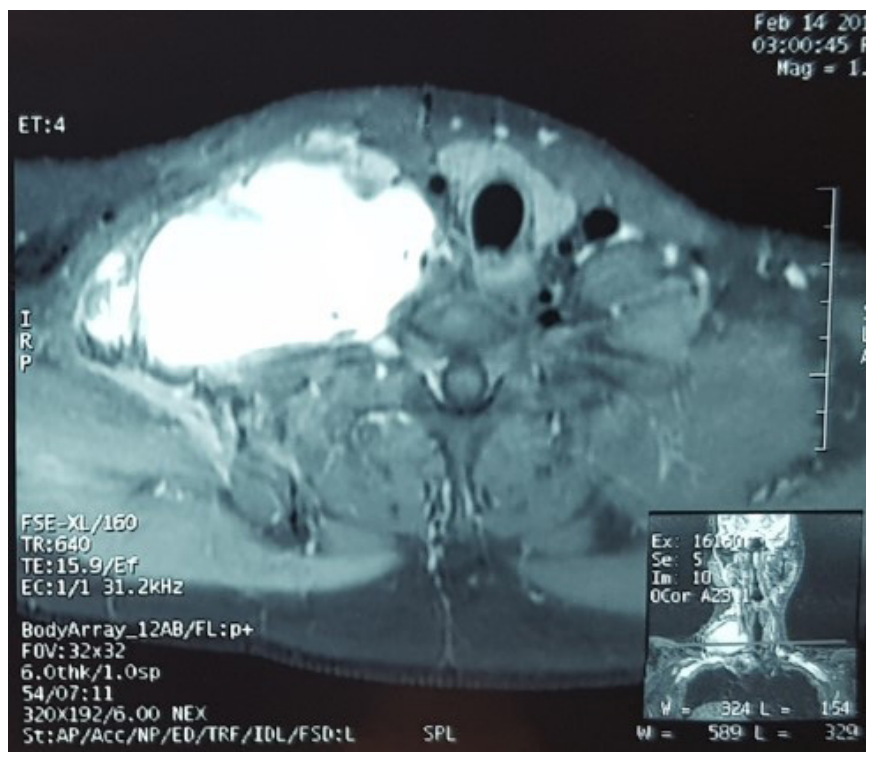

A 


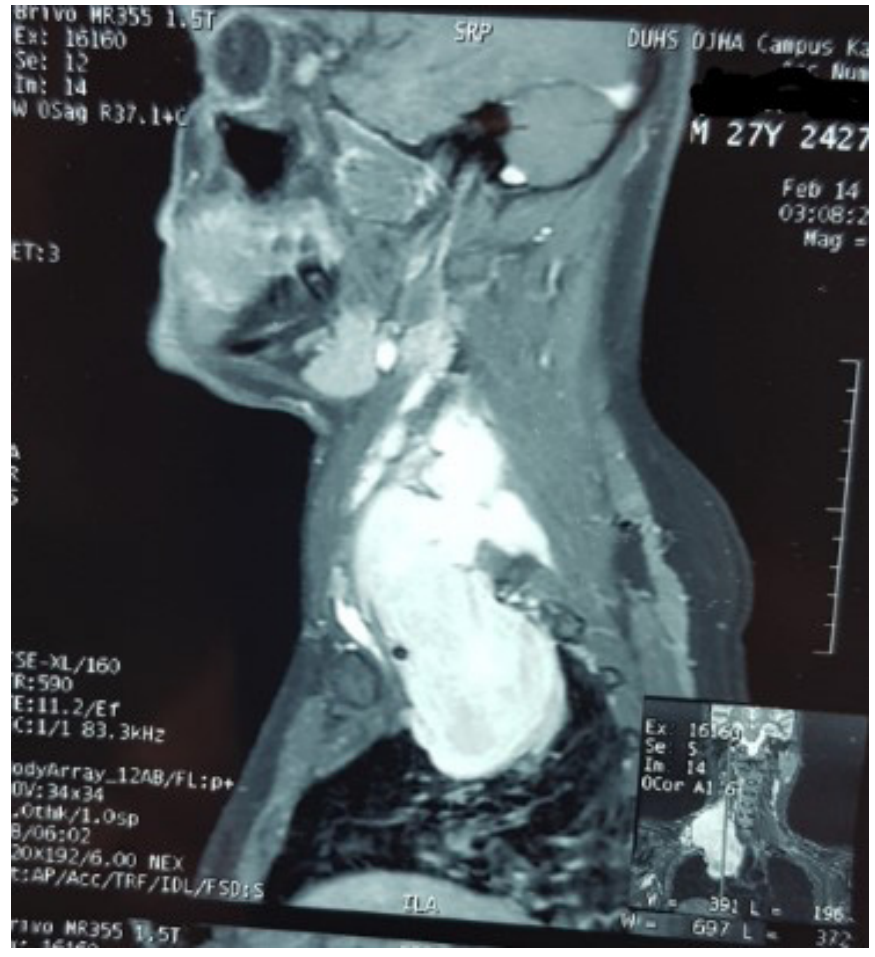

B

Fig. (1): (A) Axial \& (B) sagittal images of MRI showing tumor at the time of relapse after surgery. Abnormal signal intensity mass in the right side of neck, extending into right hemithorax.

The imaging showed marked disease progression as compared to previous (Post excision) imaging.

\section{HISTOPATHOLOGY}

Since complete surgical resection was not possible therefore, a repeat image guided biopsy of neck mass was done.

Morphologically the sections showed linear core of spindle cell lesion composed of large sweeping (A) of bland spindle shaped cells against collagenous background stroma. The cells contained indistinct cellular outlines, moderate eosinophilic cytoplasm. There was no convincing evidence of malignancy.

Immunohistochemical stains were performed and stained positive for Desmin, \& Beta Catenin, thus concurring with the diagnosis of fibromatosis.

\section{TREATMENT COURSE}

Due to aggressive and unresectable nature of disease and symptoms impacting the quality of life, the decision of starting chemotherapy was made by the multidisciplinary tumor board.

The chemotherapy with liposomal doxorubicin was initiated at dose of $50 \mathrm{mg} / \mathrm{m} 2$ every 28 days and continued for 4 cycles followed by a repeat scan that showed significant disease reduction. However, the mass was still abutting the carotid vessels and surgical excision was not possible. Therefore, the decision to continue chemotherapy was made.
Thus, the patient received 6 cycles of chemotherapy (total cumulative doxorubicin dose $300 \mathrm{mg} / \mathrm{m} 2$ ). The follow up scan was done and showed further disease reduction (Fig. 2). There was also significant subjective as well as clinical improvement in motor strength and hand gripping. However, a surgery with $\mathrm{R} 0$ resection margin was still not achievable from surgeon's perspective. The case was therefore taken to a multidisciplinary meeting for discussion.

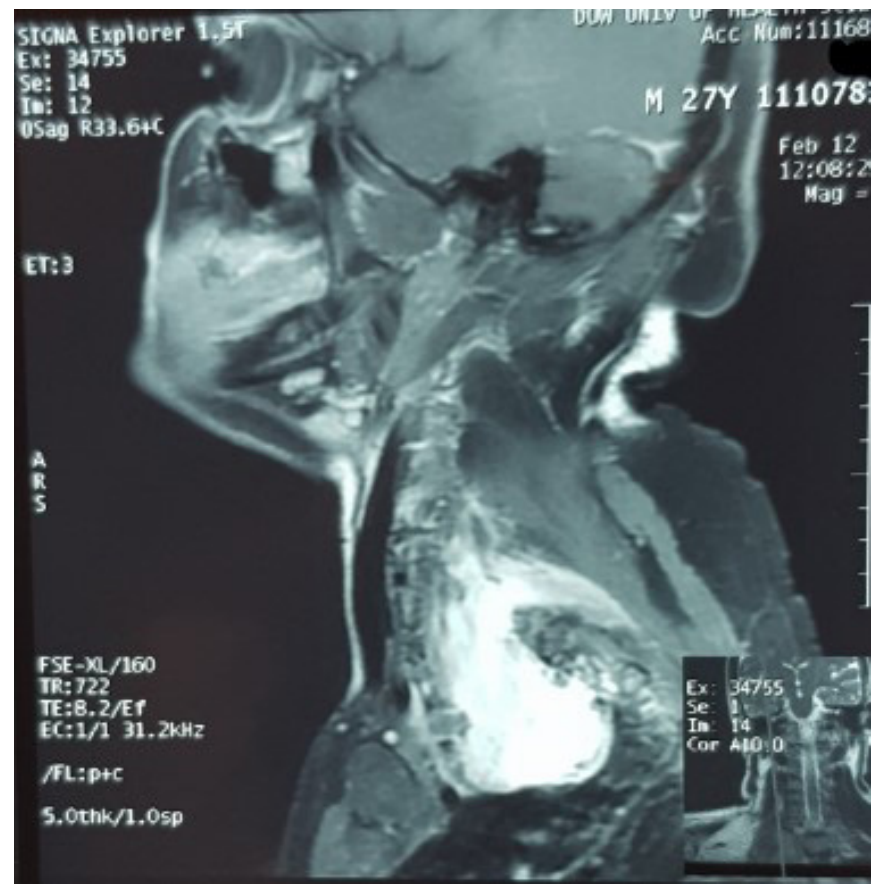

Fig. (2): MRI sagittal image of the patient after chemotherapy showing abnormal signal enhancing mass at right thoracic inlet traversing through root of neck, with radiological evidence of disease reduction, now measuring $8.4 \mathrm{~cm}$ in craniocaudal extent (previously: $14.7 \mathrm{~cm}$, refer Fig. (1A\&1B).

\section{TUMOR BOARD DISCUSSION}

In the multidisciplinary meeting, involving departments of General, Thoracic and Plastic surgery, Diagnostic and interventional radiology, Medical as well as Radiation Oncology, discussion was held on this case and due to marked improvement in quality of life, it was planned to consider maintenance treatment using non-cytotoxic agents while keeping options such as radiation as well as chemotherapy saved for progressive disease. In this regard several therapeutic options were considered, treatment recommendations reviewed, and the decision to initiate treatment, using COX 2 inhibitor, was made. The patient was kept on tab. Celecoxib at $200 \mathrm{mg}$ divided into twice daily dose after explaining the possible benefit and potential side effects for 3 months followed by reevaluation.

Upon follow up consultation via telemedicine service, beyond 2 months therapy, the patient reported remarkable clinical response, had resumed his job, and there was near complete restoration of his baseline pre disease life activities. Thus, the patient was advised to continue the treatment further and visit clinic in 1 month. 


\section{ONCOLOGIST'S PERSPECTIVE}

Desmoid tumors or aggressive fibromatosis often present difficult challenge for patients because of the extent of surgery required for optimal control, their high recurrence rate, and their long natural history. Although they do not exhibit the histopathologic features to classify them as sarcomas, desmoid tumors are often categorized as lowgrade sarcomas because of their high tendency to recur locally after excision. The primary treatment for patients with resectable desmoid tumors is surgery [6-10]. The extremity tumors, as we report, have relatively lower response and disease-free survival when compared to patients with abdominal wall tumors, with 10-year DFS rates $88 \%$ vs $62 \%$, respectively $(P<.01)[11,12]$. The impact on local control and risk of recurrence, due to positive resection, remains controversial [13]. Recent meta-analysis including data from 1295 patients, from 16 studies, found that R1 resections were associated with an almost 2-fold higher risk of recurrence [14].

Treatment choices for symptomatic patients with large tumors that cause morbidity, pain, or functional limitation, should be based on the location of the tumor and potential morbidity of the treatment. Options include surgery and/or RT and/or systemic therapy. The consensus upon optimal chemotherapy regimen for such low-grade tumors has not yet been clearly defined. However, in patients with definitely progressing tumors, the decision about systemic treatment should be individualized in a multidisciplinary setting. The options for systemic therapy such as cytotoxic chemotherapy drugs, hormonal or biological agents, or non-steroidal anti-inflammatory drugs (NSAIDs), have shown promising results in patients with desmoid tumors $[15,16]$. Doxorubicin-based chemotherapy has been effective in patients with recurrent or unresectable tumors [17-19]. The combination regimens of methotrexate with either vinorelbine or vinblastine has also been shown to be associated with prolonged stable disease in case of unresectable or recurrent tumors [20, 21].

The current guidelines have included NSAIDs (sulindac or celecoxib) as systemic therapy options for patients with advanced or unresectable desmoid tumors. Other NSAIDS such as meloxicam has also been tried and demonstrated efficacy [22]. The effectiveness of celecoxib has been proven in cases involving the use of genetic testing [23]. As well as in intra-abdominal desmoid tumors where the disease response using celecoxib after surgical resection, was demonstrable up till 2-year follow up period [24].

Considering the young age and active life style of the patient, the rationale behind using an approach of maintenance treatment was to provide maximum possible response while keeping treatment related toxicities to a minimum, and saving more invasive options such as further cytotoxic chemotherapy or radiation for future, until maximum response is achieved and clear guidelines or a more novel approach becomes available and well suited.

\section{SURGEON'S PERSPECTIVE}

Surgery can offer complete cure from localized disease. Surgery with tumor free margin (approx. $3 \mathrm{~cm}$ ) is required.

Therefore, in large fibrous tumor excision, this may result in loss of limbs and / or debilitating post-surgical deformity. All of this is tolerable if we can reasonably assure eradication of localized disease.

Frequently surgery is performed, as in this case with inadequate resection margins resulting in local recurrence of tumor. After incomplete resection and subsequent recurrence of tumor, it is rarely possible to do a Redo surgery with adequate resection margins.

However, in this case, though the disease was advanced, there was a chance to get a complete resection but this would have involved loss of upper limb. This was not acceptable to the patient; therefore, surgery was declined by the patient [25].

In such cases, if Oncology achieves regression of tumor then we can reassess for surgical resection.

\section{REFERENCES}

1. Lazar AJ, Tuvin D, Hajibashi $S$, et al. Specific mutations in the beta-catenin gene (CTNNB1) correlate with local recurrence in sporadic desmoid tumors. Am J Pathol 2008; 173: 1518-27.

2. Domont J, Salas S, Lacroix L, et al. High frequency of betacatenin heterozygous muta-tions in extra-abdominal fibromatosis: a potential molecular tool for disease manage-ment. $\mathrm{Br} \mathrm{J}$ Cancer 2010; 102: 1032-6

3. Le Guellec S, Soubeyran I, Rochaix P, et al. CTNNB1 mutation analysis is a useful tool for the diagnosis of desmoid tumors: a study of 260 desmoid tumors and 191 potential morphologic mimics. Mod Pathol 2012; 25: 1551-8.

4. Colombo C, Miceli R, Lazar AJ, et al. CTNNB1 45F mutation is a molecular prognostica-tor of increased postoperative primary desmoid tumor recurrence: an independent, mul-ticenter validation study. Cancer 2013; 119: 3696-3702.

5. Mullen JT, DeLaney TF, Rosenberg AE, et al. Beta-catenin mutation status and out-comes in sporadic desmoid tumors. Oncologist 2013; 18: 1043-9.

6. Lev D, Kotilingam D, Wei C, et al. Optimizing treatment of desmoid tumors. J Clin Oncol 2007; 25: 1785-91.

7. Pritchard DJ, Nascimento AG, Petersen IA. Local control of extraabdominal desmoid tumors. J Bone Joint Surg Am 1996; 78: 84854.

8. Ballo MT, Zagars GK, Pollack A, et al. Desmoid tumor: prognostic factors and outcome after surgery, radiation therapy, or combined surgery and radiation therapy. J Clin Oncol 1999; 17: 158-67.

9. Stojadinovic A, Hoos A, Karpoff HM, et al. Soft tissue tumors of the abdominal wall: analysis of disease patterns and treatment. Arch Surg 2001; 136: 70-9.

10. Ma D, Li S, Fu R, et al. Long-term outcomes of 47 patients with aggressive fibromatosis of the chest treated with surgery. Eur $\mathrm{J}$ Surg Oncol 2016; 42: 1693-8.

11. Gronchi A, Casali PG, Mariani L, et al. Quality of surgery and outcome in extra-abdominal aggressive fibromatosis: a series of patients surgically treated at a single insti-tution. J Clin Oncol 2003; 21: 1390-7.

12. Peng PD, Hyder $\mathrm{O}$, Mavros MN, et al. Management and recurrence patterns of des-moids tumors: a multi-institutional analysis of 211 patients. Ann Surg Oncol 2012; 19: 4036-42. 
13. Melis M, Zager JS, Sondak VK. Multimodality management of desmoid tumors: how important is a negative surgical margin? J Surg Oncol 2008; 98: 594-602.

14. Janssen ML, van Broekhoven DL, Cates JM, et al. Meta-analysis of the influence of sur-gical margin and adjuvant radiotherapy on local recurrence after resection of sporadic desmoid-type fibromatosis. Br J Surg 2017; 104: 347-57.

15. Janinis J, Patriki M, Vini L, et al. The pharmacological treatment of aggressive fibroma-tosis: a systematic review. Ann Oncol 2003; 14: $181-90$

16. de Camargo VP, Keohan ML, D'Adamo DR, et al. Clinical outcomes of systemic therapy for patients with deep fibromatosis (desmoid tumor). Cancer 2010; 116: 2258-65.

17. Poritz LS, Blackstein M, Berk T, et al. Extended follow-up of patients treated with cyto-toxic chemotherapy for intra-abdominal desmoid tumors. Dis Colon Rectum 2001; 44: 1268-73.

18. Garbay D, Le Cesne A, Penel N, et al. Chemotherapy in patients with desmoid tumors: a study from the French Sarcoma Group (FSG). Ann Oncol 2012; 23: 182-6.

19. Constantinidou A, Jones RL, Scurr M, et al. Pegylated liposomal doxorubicin, an effec-tive, well-tolerated treatment for refractory aggressive fibromatosis. Eur J Cancer 2009; 45: 2930-4.
20. Azzarelli A, Gronchi A, Bertulli R, et al. Low-dose chemotherapy with methotrexate and vinblastine for patients with advanced aggressive fibromatosis. Cancer 2001; 92: 1259-64.

21. Park KH, Choi YJ, Kim KW, et al. Combination chemotherapy with methotrexate and vinblastine for surgically unresectable, aggressive fibromatosis. Jpn J Clin Oncol 2016; 46: 845-9.

22. Nishida $Y$, Tsukushi S, Shido $Y$, et al. Successful treatment with meloxicam, a cyclooxy-genase-2 inhibitor, of patients with extraabdominal desmoid tumors: a pilot study. J Clin Oncol 2010; 28(6): e107-e109.

23. Yang $\mathrm{S}$, Wang $\mathrm{X}$, Jiang $\mathrm{H}$, et al. Effective treatment of aggressive. Fibromatosis with celecoxib guided by genetic testing. Cancer Biol Ther 2017; 18(10): 757-60.

24. Wang Y, Wong J. Complete remission of pancreatic head desmoid tumor treated by COX-2 inhibitor-a case report. World J Surg Onc 2016; 14: 190.

25. Smith SC, Gooding WE, Elkins M, et al. Solitary fibrous tumors of the head and neck: a multi-institutional clinicopathologic study. Am J Surg Pathol 2017; 41(12): 1642-56. 